\title{
LA CULTURA ARBITRARIA Operativización del concepto
}

\author{
RAFAEL GOBERNADO ARRIBAS
}

Universidad de Málaga

PALABRAS CLAVE ADICIONALES

Polarización, Valores.
ADDITIONAL KEYWORDS

Polarization, Values.

RESUMEN. Se entiende por cultura arbitraria aquella que sirve para distinguirse e identificarse. La arbitrariedad se aprecia en cualquier elemento cultural: los valores, las normas, las ideas, los símbolos y la cultura material. La Encuesta Mundial sobre Valores de 1996 nos ha permitido indagar operativamente sobre el aspecto arbitrario de los valores. La arbitrariedad en los mismos se aprecia en la polarización de las respuestas. Un indicador de polarización es el coeficiente de curtosis. Según lo observado, la polarización es un rasgo propio de las respuestas a preguntas sobre valores.

ABSTRACT. We understand that the phrase 'arbitrary culture' serves to distinguish and identify itself. Arbitrariness can be identified in whatever element: values, rules, ideas, symbols and material culture. The 1996 World Survey of Values has allowed us to investigate effectively the arbitrary nature of values. Their arbitrariness can be seen in the polarization of the answers. An indicator of polarization is the coefficient of 'curtosis'. According to observations, polarization is a distinctive feature of answers to questions about values.

E-mail: gobernado@uma.es

Revista Internacional de Sociología (RIS)

Tercera Época, No 37, Enero-Abril, 2004, pp. 29-46. 


\section{OBJETIVOS Y CONCEPTUALIZACIÓN}

El objetivo de este trabajo es constatar empíricamente el funcionamiento de los aspectos arbitrarios de la cultura. Entendemos por cultura al conjunto de todos los elementos ideacionales del comportamiento humano. Asimismo, se supone que en la cultura hay aspectos cognitivos, arbitrarios y de coherencia interna'. Se corresponde esta clasificación con la que en su momento hizo Parsons (1976:18 y 19): elementos cognitivos, catéticos (o expresivos) y evaluadores. A su vez, la distinción entre aspectos cognitivos y arbitrarios está en deuda con la ya clásica de Durkheim (1982) entre lo profano y lo sagrado 2 . Cada uno de esos elementos ha de entenderse como abstracción de una realidad compleja e interpenetrada, ya que todo comportamiento social incorpora a la vez aspectos culturales cognitivos, arbitrarios y coherentes, en una proporción u otra.

Ahora sólo nos interesan los aspectos arbitrarios de la cultura. Bourdieu y Passeron (1981:48) afirmaban que la cultura “ [...] es arbitraria en tanto que la estructura y las funciones de esta cultura no pueden deducirse de ningún principio universal, físico, biológico o espiritual, puesto que no están unidas por ningún tipo de relación interna a la "naturaleza de las cosas'o a una 'naturaleza humana"'3. Si no está ligada a la naturaleza de las cosas o a la naturaleza humana, esa faceta de la cultura es inaprehensible de forma empírica. El funcionamiento de ese elemento arbitrario sería caótico, aleatorio e imprevisible (Schmid, 1992; Eisenstadt, 1992). Sin embargo, cabe la posibilidad de encontrar cierto orden en el mismo.

Las claves fundamentales que explican el funcionamiento de la cultura arbitraria son de dos tipos (Gobernado, 2002:879-881). El primero, se encuentra en la propia cultura objetivada, en su mapa de clasificaciones. La cultura, en su aspecto arbitrario, proporciona siempre alternativas opuestas, contradictorias: ninguna de esas alternativas está justificada atendiendo a la naturaleza de las cosas. El mapa de los aspectos arbitrarios de la cultura se organiza siguiendo el criterio de contradicción, de forma que siempre ofrezca una doble posibilidad: algo y su contrario. El mapa de clasificaciones arbitrarias es fundamentalmente una creación cultural y relativamente independiente del resto de componentes culturales y no culturales que tienen que ver con el comportamiento humano.

\footnotetext{
' El marco teórico que se expone bajo este primer epígrafe se desarrolla más ampliamente en Gobernado (2002a y 2002b).

${ }^{2}$ Véase el desarrollo teórico posterior de esta clasificación de Durkheim en Alexander (1989). Sobre el aspecto evaluativo o coherencia cultural véase Smelser (1992).

${ }^{3} \mathrm{La}$ ortografia es un claro ejemplo de arbitrariedad cultural en muchos casos. Así lo hizo ver Veblen con la ortografia inglesa, Weber con la china y el propio Bourdieu con la francesa.
} 
El segundo tipo se encuentra en los actores sociales inmersos en esa cultura. Se trata de la necesidad sentida por los actores de identificación y distinción. Es una doble necesidad complementaria: constituyen la cara y la cruz de una misma moneda. El actor, dice Gobernado (2003), "pretende mediante su comportamiento un doble objetivo: identificarse con otros iguales y, al mismo tiempo, distinguirse de los demás. El proceso se produce tanto entre individuos como entre colectivos; tanto en el nivel micro social, como en el macro social. Se produce y reproduce constantemente". La identificación y la distinción se producen, además, entre actitudes radicales y actitudes moderadas a la hora de aceptar un mismo elemento cultural. Este doble proceso de identificación y distinción es el que proporciona la apariencia de caos y aleatoriedad en la cultura arbitraria. En ningún colectivo humano puede haber acuerdo claro sobre cualquier aspecto arbitrario de la cultura, ya que el desacuerdo con los demás individuos, categorías sociales o grupos ha constituido siempre un buen mecanismo de distinción ${ }^{4}$.

La cultura arbitraria, por lo tanto, es el medio idóneo para que tenga lugar el doble proceso de identificación y distinción. La razón de ser de la existencia de la arbitrariedad cultural, junto con la necesidad de identificación y distinción, es crear diversidad cultural (Gobernado, 2002 y 2003). La noción de diversidad cultural está muy próxima a la de biodiversidad: posiblemente se han adaptado mejor aquellos colectivos que incorporaban suficiente diversidad cultural que aquellos otros que eran culturalmente homogéneos.

La arbitrariedad cultural se aprecia en cualquiera de los elementos que componen la cultura: tanto en los valores como en las normas, creencias, conocimientos (incluso), símbolos y cultura material. Sin embargo, seguramente son los valores los que mejor responden a los criterios de arbitrariedad que hemos apuntado arriba. Los valores son criterios de preferibilidad (Williams, 1979: 16; Del Pino Artacho y Bericat, 1998:2) que por definición siempre oscilan entre dos posiciones contrarias (Ferrater Mora, 1979: 3376; Marías, 1980:408; Frondizi, 1972: 19; Williams, 1977). Todas las definiciones de los valores destacan la polaridad que suponen: lo feo frente a lo bello; lo justo frente a lo injusto; lo bueno frente a lo malo, etc. Para un repaso de diversos mapas de valores polarizados véase cualquiera de los estudios empíricos sobre los mismos (Díez Nicolás e Inglehart, 1994; Rokeach, 1979; Orizo, 1996; Kaireo, 1994; Bericat, 1989; Inglehart, 1991; López Casero et al., 1994; Torcal, 1992, etc.). Para un buen resumen de mapas valorativos polarizados, desde un punto de vista teórico, véase Ros (2002).

\footnotetext{
${ }^{4}$ Sobre distinción e identificación véase, entre otros: Bourdieu (1988); Boudon (1981:173-180); Veblen (1974); Alexander (1988); Valcarcel (1995); Javaloy et al., (2001:291-344); Luhmann (1998a: 31-57 y 1998b:71-98).
} 


\section{RIS}

De acuerdo con el párrafo anterior, los valores proporcionan el aspecto arbitrario idóneo para que se ejercite la identificación y la distinción por parte tanto de los individuos como de los grupos, las categorias sociales y demás niveles de sociabilidad. Las respuestas a las preguntas sobre valores deben, según esto, ofrecer un aspecto polarizado. Se entiende por polarización la tendencia a ubicarse en los extremos de la distribución ${ }^{5}$.

La polarización, que resulta ser el tema básico ahora, no ha sido resaltada habitualmente por los estudios empíricos sobre adscripción a valores, pese al interés despertado en los estudios teóricos. Solamente se han utilizado los valores extremos de las respuestas para calcular indirectamente la carga emocional (véase por ejemplo Del Pino Artacho y Bericat, 1998:33) que acompaña a las mismas. O sea, pese a observar la polarización correspondiente, no se ha dado importancia a la misma.

Posiblemente tal desinterés por la polarización sea propio de la mayoría de los análisis sociológicos que olvida el conflicto. Todo lo contrario ocurre en los análisis de ciencia política y, en particular, en los estudios sobre opinión pública, en los que se dedica mucha atención a procesos como los de polarización, fragmentación y división ${ }^{6}$. La idea de ruptura, o línea de separación entre dos extremos o polos, es habitual en este tipo de análisis (véase, por ejemplo, Torcal y Chhibber, 1995). Precisamente, ha sido en este tipo de estudios donde hemos encontrado la forma de operativizar el concepto de polarización, concretamente en DiMaggio, Evans y Bryson (1996). Téngase en cuenta que las respuestas sobre valores difieren claramente de las respuestas sobre opinión pública: la opinión pública habitualmente no implica enfrentamiento de opiniones sobre un asunto (distribución en forma de "U"), sino consenso sobre el tema (distribución normal o en forma de "J" o "L") (véase Sierra Bravo, 1998:388); mientras que las respuestas sobre valores están mayoritariamente polarizadas, como luego se verá.

En el artículo citado de DiMaggio et al. (1996: 694-697), los autores aprecian cuatro dimensiones en el concepto de polarización ${ }^{7}$ pero a nosotros sólo nos interesa una de ellas, la bimodalidad. La opinión pública está polarizada en la medida

\footnotetext{
${ }^{5}$ Escobar (1998:10) llama la atención sobre tres dimensiones de la diversidad que pueden llegar a confundirse: La desviación o distancia con respecto a un punto de referencia (en general, la media aritmética); la desigualdad o distancia recíproca entre todos los sujetos de la población; y la polarización o tendencia a ubicarse en los extremos de la distribución.

${ }^{6}$ Un ejemplo que sirve como excepción, que se encuentra a medio camino entre la ciencia politica y el análisis sociológico de la cultura, es el libro editado por Berger (1999), en el que la idea de polarización y conflicto cultural es central.

${ }^{7}$ Las cuatro dimensiones de polarización son las siguientes: 1) la dispersión, medida por la varianza; 2) la bimodalidad, medida por el coeficiente de curtosis; 3 ) la coherencia, medida por el coeficiente alfa de Cronbach, y 4) la consolidación, medida por los análisis de medias entre grupos e intra grupos.
} 
en que las respuestas sobre una misma pregunta se agrupan en campos separados, ubicadas entre dos posiciones modales. Hay que destacar que la bimodalidad es diferente de la distancia entre respuestas. Lo que arriba señalábamos como carga emocional tiene más que ver con la distancia entre las respuestas que con la bimodalidad. El indicador de bimodalidad es el coeficiente de curtosis o apuntamiento. Indica en qué medida la distribución se acumula alrededor de un valor o alrededor de varios. Aunque la curtosis se utiliza habitualmente como un indicador de diagnóstico, en este caso el interés es directo sobre ella. El valor de la curtosis es positivo cuando existe un amplio nivel de consenso (la representación gráfica de la distribución es más apuntada que la distribución normal); es negativo, cuando el consenso es menor (su representación gráfica es más plana que la distribución normal). Toma el valor cero cuando la distribución presenta el mismo perfil que la normal correspondiente. La bimodalidad en sentido estricto se produce a medida en que el valor se aproxima a -2 (véase, por ejemplo Fernández-Abascal et al., 1994:274) ${ }^{8}$.

La curtosis como indicador de bimodalidad está suficientemente acreditada (Chissom, 1970; Darlington, 1970; Smith, 1991). Cuanto más alto sea el valor negativo de la curtosis, mayor polarización se encuentra en las respuestas. La máxima polarización posible entre individuos, siguiendo los múltiples criterios de polaridad (tanto entre los polos de la valoración, como entre radicalismo y moderación a la hora de asumir los valores), se produce cuando la curtosis es 1,3 negativa; es decir, se aprecia en aquella distribución de datos cuya representación gráfica es plana. Cualquier otro valor negativo superior de la curtosis indica que la elección de valores está condicionada por categorías sociales propias de la estructura social (clases sociales, sexo, grupos de edad, relaciones ocupacionales, etc.). La polarización se aprecia además por el hecho de que la curtosis dentro de cada una de las posibles categorías sociales es también negativa.

De acuerdo con lo anterior, la hipótesis principal de este trabajo puede operativizarse en los siguientes términos: las respuestas a preguntas sobre valores presentarán coeficientes de curtosis negativos en mayor proporción que las respuestas a preguntas que no sean sobre valores.

\footnotetext{
${ }^{8}$ La fórmula del coeficiente de curtosis es:

$\mathrm{k}=\left\{\left[\Sigma(\mathrm{X}-\mathrm{m})^{4}: \mathrm{N}\right] / \mathrm{s}^{4}\right\}-3$

En donde $m$ es la media y $s$ es la desviación estandar.
} 


\section{EL MÉTODO}

Para revalidar la hipótesis anterior, hemos utilizado los datos de la Encuesta Mundial de Valores de 1995-96 sobre cuarenta y nueve países o territorios, en particular, los datos de la Encuesta de Valores de Andalucía de 1996 (Del Pino Artacho y Bericat, 1998), los de España y los de Estados Unidos. De toda la información disponible hemos restringido nuestro interés a un conjunto de preguntas del cuestionario dirigidas a descubrir las preferencias sobre la organización de la sociedad (estas preferencias reciben habitualmente el nombre de ideología). En total son seis preguntas que dan a escoger a los encuestados una respuesta entre dos alternativas extremas: una sociedad organizada según criterios de mercado frente a una sociedad organizada de forma planificada por el Estado (desde la variable v125 a la v130 inclusive, del cuestionario de 1996) ${ }^{9}$. Estas preguntas organizan sus respuestas en un rango de diez puntos: 0 para los defensores acérrimos de la planificación estatal y 10 para los correspondientes del libre mercado. Los encuestados escogen la respuesta (el número) que se aproxima más a su ideología.

La manera de resaltar las particularidades de las respuestas a preguntas sobre valores consiste en contrastarlas con otras respuestas a preguntas no valorativas, sino de tipo cognitivo pero de semejante sintaxis. Hemos escogido dos preguntas que reunen ambas características: no se refieren a valores y tienen semejante sintaxis, es decir, presentan un rango de diez puntos como opciones de respuesta; y otra tercera pregunta que, aunque no presente una sintaxis idéntica, admite fácilmente la comparación. Una de ellas es la que inquiere por los ingresos familiares (v227); otra, es la que inquiere por el grado de satisfacción respecto a la vida del propio entrevistado (v65); la tercera pregunta se refiere a la edad en que se terminó la escolaridad (v218).

La pregunta sobre ingresos es una pregunta de hecho. Hace referencia a una realidad objetiva y es, o pudiera ser, contrastable. El entrevistado podrá equivocarse o mentir, pero hay una realidad externa que nos puede permitir afirmar si es verdadera o falsa. El inconveniente que plantea la pregunta sobre ingresos en nuestro análisis es la posibilidad de que las respuestas adopten cierta polarización proveniente del particular reparto de las rentas en las sociedades modernas. Las hipótesis deducidas de las ideas de "sociedad dual" de André Gorz y de "estructura dual de trabajo" de Pioré, así lo parecen apuntar.

\footnotetext{
${ }^{9}$ La razón de esta selección se encuentra en la idoneidad de tales preguntas para el tipo de análisis que nos traemos entre manos. Son preguntas cuyas respuestas encajan tanto en la cultura arbitraria, como en la cultura cognitiva. De la misma forma, las respuestas también dependen de los intereses de los encuestados en función de sus posiciones sociales. En la medida en que son varias preguntas sobre temas variados pero lógicamente relacionadas, permiten el análisis sobre coherencia cultural de los entrevistados. Además, las posibilidades de respuesta se presentan en un abanico cuyo rango es de diez puntos, lo que permite acentuar la polaridad si la hubiera.
} 
La pregunta sobre la satisfacción con la vida tampoco se refiere a valores. En principio, hace también referencia a una realidad objetiva y no opinable. No obstante, a la posibilidad de un reparto polarizado de la satisfacción entre la población, hay que añadir cierta proporción de arbitrariedad en la respuesta. La sintaxis de esta pregunta es la misma que la de las preguntas sobre valores aquí consideradas.

La pregunta sobre la edad en que se terminó la escolaridad es una típica pregunta de hecho, y sobre la que no cabe arbitrariedad en la respuesta. En la medida en que se refiere a un indicador de desigualdad social, pudiera adquirir la forma polarizada al igual que la distribución de ingresos, aunque los datos no lo han apreciado. Tiene el inconveniente de que no reviste la sintaxis de las preguntas sobre valores. Se trata de una pregunta abierta en la que cada encuestado pone directamente la edad en la que terminó los estudios.

A lo largo del trabajo hemos utilizado como criterio de significación de relaciones entre las variables la prueba de la F (Sanchez Carrión, 1995:339-341), propia para cuando se trabaja con medias en la variable dependiente, y la variable independiente es cualitativa. En resumen, consiste en comparar la varianza entre grupos (o varianza explicada) y la varianza intra grupos (o varianza no explicada). Se ha de advertir, por último, que hemos seguido el mismo criterio en la agrupación de los valores de las variables que el utilizado en el estudio matriz de esta investigación (Pino y Bericat, 1998: 329-331) ${ }^{10}$.

\footnotetext{
${ }^{10}$ La principal crítica que se puede hacer al método seguido aquí es que la propia forma de la pregunta sobre valores induce a la polarización. Es decir, en la medida en que hay que señalar una preferencia entre dos extremos el resultado normal es la polarización. Sin embargo esto no es del todo cierto. El tipo de escala Cantril utilizado en la encuesta (elegir entre 10 posibilidades, por ejemplo desde el 1 -extrema izquierda- al 10 -extrema derecha-) atenúa tal posibilidad. Cuando la pregunta se dirige a inquirir por temas no directamente valorativos, como es el caso de optar entre derecha o izquierda, la distribución de las respuestas se suele agrupar en el centro.

La razón contraria se puede esgrimir sobre la variable "renta". Se entiende en este caso que por definición la renta no tiende a polarizarse. Pero tampoco es del todo cierta esta observación. El mercado laboral español, por ejemplo, está dividiéndose entre "estables" y "precarios" (J.G. Polavieja, 2003:136-153) con sueldos diferentes según categorías ocupacionales. $O$ sea, la polarización no es algo ajeno a la distribución de la renta. De hecho en la encuesta utilizada en este trabajo son muchos los países que presentan polarización en su distribución de la renta.

Además, hay que aceptar la idea de que la polarización (o distinción) tiene que ver con los límites del sistema elegido. Así, por ejemplo, la polarización puede darse frente a países vecinos, con lo que en el interior del país la aceptación del valor correspondiente será casi unánime.

Por último, aunque se debería dar por supuesto, el modelo de distinción versus identificación sólo explica parte del comportamiento cultural arbitrario, no puede agotar por completo su explicación. Siempre queda una parte inexplicada debida a la interacción entre los demás aspectos culturales y no culturales. Y siempre queda la posibilidad también de que haya algo más que la distinción y la identificación, detrás de la arbitrariedad cultural.
} 


\section{RESULTADOS GLOBALES}

La Encuesta Mundial de Valores de 1996, de cuyos datos disponemos en estos momentos, ha sido aplicada en 49 territorios: Estados y regiones. De ellos, 12 territorios presentan una distribución de ingresos con curtosis positiva, es decir, con distribución no polarizada (dos países no ofrecen datos sobre ingresos); 43 territorios presentan una distribución de edades en que se termina la escolaridad con curtosis positiva (dos países no ofrecen datos sobre la edad de abandono de la escolaridad); igualmente, 32 territorios presentan una distribución sobre la satisfacción con la vida con curtosis positiva. O sea, en la mayoría de los países no se aprecia polarización en dos de las tres variables que no se refieren a valores. En la otra variable, la de los ingresos, se aprecia polarización en cierto número de países, pero no con la suficiente insistencia como para pensar que constituye un rasgo propio de esa variable.

Otra cosa distinta ocurre con los valores. Para facilitar la comparación, hemos limitado la observación a dos preguntas sobre valores: una es la que da a escoger entre igualdad de salarios frente a incentivos laborales (v125) y la otra, es la que da a escoger entre incremento de la propiedad privada de las empresas frente a incremento de la propiedad estatal de las empresas (v126). En ambos casos la curtosis es claramente negativa, es decir, existe polarización. La distribución de las respuestas de la primera variable (v125) presenta curtosis negativa en 47 de los 49 territorios encuestados; la segunda variable sobre valores (v126) presenta una distribución con curtosis negativa en 43 territorios.

Los datos sobre Andalucía de la Encuesta de Valores de 1996 mantienen la misma tónica. $\mathrm{Al}$ trabajar con una sola región podemos observar más fácilmente las distribuciones de las seis variables referidas a valores. La curtosis es negativa en todos los casos. Incluso en algunos se acerca a una acusada polarización: en la pregunta (v125) sobre igualdad de ingresos frente a incentivos laborales $(\mathrm{k}=$ -1,419); en la elección (v130) entre la creencia de que las personas sólo pueden hacerse ricos a costa de otros frente a la creencia en que es posible crear riqueza sin quitársela a nadie $(\mathrm{k}=-1,241)$; y en la elección ( $\mathrm{v} 126)$ entre propiedad privada frente a propiedad estatal $(\mathrm{k}=-1,084)$.

\section{ARBITRARIEDAD FRENTE A INTERÉS}

Cuando es el interés el que condiciona la opción ideológica (Perry, 1954; Boudon, 1979 y 1981), las ideologías de las categorías sociales, en especial si las categorías son ocupacionales, difieren claramente entre sí: no pueden desear la misma organización social los empresarios que los asalariados, los trabajadores manuales que los no manuales, los que ganan mucho dinero que los que ganan poco, etc. Además, esto implica que dentro de cada categoría social los valores tienden a 
agruparse alrededor de la media (curtosis positiva), es decir, son categorías internamente más homogéneas que la sociedad en la que se desarrollan.

Sin embargo, los datos informan que la estructura social (léase el interés) explica en muy pequeña proporción la varianza de opciones ideológicas (Kerhofs, 1994: 47). Tiene más importancia explicativa la hipótesis de la polarización. Para facilitar el análisis que sigue se ha trabajado con la pregunta (v125) sobre valores: escoger entre igualdad de salarios versus incentivos salariales.

De entrada, las medias de las respuestas (v125) entre los cuarenta y nueve países encuestados difieren poco entre sí. La peor o mejor situación económica (la media de ingresos) justifica en parte tales escasas diferencias. De cualquier forma, la similitud entre las medias de los diferentes países obliga a pensar que los intereses explican poco la adscripción a valores.

Pero hay más: la polarización sigue siendo evidente en las valoraciones según las distintas categorías sociales que conforman la estructura social. Nos limitamos ahora a los datos de Andalucía.

A) Según niveles de ingresos. Los ingresos (tabla 1) apenas llegan a explicar un tres por ciento de la varianza de esa pregunta. Pues bien, las respuestas a las preguntas sobre valores proporcionan una curtosis negativa en las tres categorías de ingresos. Incluso en el nivel de ingresos inferior, la curtosis se acerca más a la polaridad $(\mathrm{k}=-1,41)$.

B) Según tipos de profesión. Las diferencias entre las medias de ingresos de las ocupaciones manuales y no manuales es amplia $(\mathrm{F}=186,1 ;$ sig. $=0,000)$ (tabla 2). Las ocupaciones no manuales reciben mayores ingresos, como se supone. Tanto en el total como en cada una de las categorías, la curtosis es ahora positiva. Podría esperarse que esta "racionalidad" salarial entre categorías se plasmara en otra "racionalidad" ideológica equivalente, pero no es así. La relación entre

Tabla 1.

v125 según categorias de ingresos (Andalucia).

\begin{tabular}{lllll}
\hline Ingresos & Media & Varianza & Curtosis & $\mathrm{n}$ \\
\hline Bajos & 4,77 & 10,76 & $-\mathbf{1 , 4 1}$ & 711 \\
Medios & 5,4 & 9,7 & $\mathbf{- 1 , 3 6}$ & 467 \\
Altos & 6,34 & 7,77 & $\mathbf{- 0 , 8 3}$ & 164 \\
Total & 5,18 & 10,28 & $\mathbf{- 1 , 4 2}$ & 1342 \\
\hline
\end{tabular}

Fuente: Encuesta de Valores de Andalucía, 1996 (elaboración propia).

$\mathrm{F}=18,2 ;$ Sig. $=0,000 ;$ Coef. de determinación $=3 \%$ 
Tabla 2.

Ingresos según tipo de profesión (no manual / manual) (Andalucia).

\begin{tabular}{lllll}
\hline $\begin{array}{l}\text { Tipo de } \\
\text { profesión }\end{array}$ & Media & Varianza & Curtosis & $\mathrm{n}$ \\
\hline No manual & 4,61 & 3,42 & $\mathbf{0 , 0 7}$ & 309 \\
Manual & 3,19 & 1,91 & $\mathbf{2 , 4 9}$ & 740 \\
Total & 3,61 & 2,76 & $\mathbf{1 , 3}$ & 1049 \\
\hline
\end{tabular}

Fuente: Encuesta de Valores de Andalucía, 1996 (elaboración propia). $\mathrm{F}=186,1 ;$ Sig. $=0,000$

Tabla 3.

v125 según tipo de profesión (no manual / manual) (Andalucía).

\begin{tabular}{lllll}
\hline $\begin{array}{l}\text { Tipo de } \\
\text { Profesión }\end{array}$ & Media & Varianza & Curtosis & $\mathrm{n}$ \\
\hline No manual & 5,64 & 9,31 & $\mathbf{- 1 , 2 7}$ & 437 \\
Manual & 4,81 & 11,1 & $\mathbf{- 1 , 4 3}$ & 865 \\
Total & 5,1 & 10,63 & $\mathbf{- 1 , 4 4}$ & 1302 \\
\hline
\end{tabular}

Fuente: Encuesta de Valores de Andalucía, 1996 (elaboración propia).

$F=19,14 ;$ Sig. $=0,000$

C) Según tipo de relación laboral. La media de ingresos de los trabajadores por cuenta propia son mayores que la de los que trabajan por cuenta ajena (véase tabla $\mathrm{n}^{\circ} 4$ ). La curtosis es positiva en los ingresos por cuenta ajena y negativa en los ingresos por cuenta propia. La correspondencia entre tipo de relación laboral y media de ingresos es grande $(\mathrm{F}=104,7 ; \mathrm{sig} .=0,000)$. Esta desigualdad de ingresos tampoco se traduce en otra desigualdad ideológica consecuente. La correspondencia entre ideología y tipo de relación laboral es también menor $(\mathrm{F}=10,17 ;$ sig. $=0,001)$ que antes $\mathrm{y}$, además, la curtosis se presenta negativa en las dos categorías ocupacionales observadas (tabla 5).

El coeficiente de curtosis de la distribución de las preguntas sobre valores sigue siendo negativo con cualquier otra clasificación que se utilice, y en todas las categorías de cada clasificación: por grupos de edad (jóvenes, adultos, mayores) (polarización que no ocurre con la correspondiente distribución de ingresos); 
Tabla 4.

Ingresos según tipo de relación laboral (cuenta propia / ajena) (Andalucía).

\begin{tabular}{lcccc}
\hline $\begin{array}{l}\text { Relación } \\
\text { Laboral }\end{array}$ & Media & Varianza & Curtosis & $\mathrm{n}$ \\
\hline Cuenta propia & 4,87 & 4,27 & $\mathbf{- 0 , 3 7}$ & 143 \\
Cuenta ajena & 3,41 & 2,24 & $\mathbf{1 , 5}$ & 906 \\
TOTAL & 3,61 & 2,76 & $\mathbf{1 , 3}$ & 1049 \\
\hline
\end{tabular}

Fuente: Encuesta de Valores de Andalucía, 1996 (elaboración propia) $\mathrm{F}=104,7 ;$ Sig. $=0,000$

Tabla 5.

v125 según tipo de relación laboral (cuenta propia / ajena) (Andalucia).

\begin{tabular}{lcccc}
\hline $\begin{array}{l}\text { Relación } \\
\text { Laboral }\end{array}$ & Media & Varianza & Curtosis & $\mathrm{n}$ \\
\hline Cuenta propia & 5,78 & 8,72 & $\mathbf{- 1 , 2 7}$ & 192 \\
Cuenta ajena & 4,97 & 10,7 & $\mathbf{- 1 , 4 3}$ & 1110 \\
TOTAL & 5,1 & 10,63 & $\mathbf{- 1 , 4 4}$ & 1302 \\
\hline
\end{tabular}

Fuente: Encuesta de Valores de Andalucía, 1996 (elaboración propia). $\mathrm{F}=10,17$; Sig. $=0,001$

sexo; actividad o inactividad laboral; el nivel de estudios (sin estudios, primarios, medios, superiores) (a diferencia de la correspondiente distribución de ingresos cuyas curtosis son todas positivas); y el tamaño poblacional del hábitat (pequeños, medianos, grandes) (a diferencia también de la correspondiente distribución de ingresos cuyas curtosis son todas ellas positivas).

Solamente hemos descubierto una categoría en la que la curtosis ideológica fuera positiva: la de la clase social subjetiva "alta". Aunque en este caso la explicación puede venir del hecho de que el número de encuestados que se autoclasificó en esa categoría es muy bajo. En las demás categorías de clase subjetiva la curtosis correspondiente es negativa. La curtosis de la clase social "baja" alcanza incluso un valor acusado de polarización $(\mathrm{k}=-1,561)$.

De acuerdo con los datos y con lo esperado (Kerhofs, 1994:47), la estructura social influye muy poco en la selección de la respuesta. En ningún momento 
RIS

REVISTA INTERNACIONAL DE SOCIOLOCIA

No 37, ENERO-ABRIL, 2004

RAFAEL GOBERNADO ARRIBAS

se encuentra la línea de fractura del ámbito social que explique la elección de una respuesta $u$ otra a las preguntas sobre valores, a diferencia de lo que suele ocurrir con otro tipo de preguntas. Además, y esto es lo importante, en cada una de las categorías propias de las variables independientes, se vuelve a reproducir los resultados polarizados de la variable dependiente (v125). La polarización se mantiene de forma permanente. De acuerdo con ello, los intereses emanados de las posiciones sociales parecen tener mucho menos peso que la presión que empuja hacia la polarización. Parece evidente, a partir de estos datos, que la curtosis negativa es una cualidad propia de la distribución de las preguntas sobre valores; mientras que la distribución de preguntas de otro tipo suele adoptar una curtosis positiva en mayor número de ocasiones. En resumen, se aprecia una clara tendencia a la polarización en las respuestas sobre valores.

\section{POLARIZACIÓN Y NIVELES DE SOCIABILIDAD}

Tal y como se dijo al principio, la identificación y la distinción se producen en todos los niveles de sociabilidad, tanto en el nivel cotidiano entre individuos, como en el nivel macro entre países, clases y categorías sociales (por ejemplo, entre hombres y mujeres, entre generaciones) y en el nivel intermedio u organizacional. La hipótesis secundaria que defendemos bajo este epígrafe es la siguiente: a medida que la sociedad está más desarrollada (o es más moderna) la identificación y la distinción se producen más abundantemente en el nivel cotidiano y menos en el nivel macrosocial.

La razón de ello se encuentra en el hecho de que cuanto más desarrollado esté un país, menos explican las clases y las categorías sociales el comportamiento de los actores. Este hecho se relaciona a su vez con tres procesos sociales típicos de las sociedades desarrolladas: mayor número de posiciones sociales en la estructura social; mayor número de posiciones sociales a las que suele pertenecer un mismo actor; aumento de la movilidad entre esas posiciones (Durkheim, 1973; Eisenstadt, 1992; Smelser, 1971; Treiman, 1970, etc). Por todo ello, la clase y las categorías sociales de pertenencia pierden interés como instrumento de identificación y distinción. En otras palabras, la cultura arbitraria se independiza aún más de la estructura de posiciones sociales.

El método para verificar esta hipótesis secundaria es simple: cuanto menor es la relación entre la cultura arbitraria y la estructura de posiciones sociales, más dependerá la cultura arbitraria de la interacción cotidiana, en el caso de que se mantenga constante la polarización.

El análisis comparado de los datos de Estados Unidos y España es muy útil para contrastar dos etapas diferentes de modernización. La idea que se mantiene ahora es que el desarrollo rompe las rigideces de la estructura social. En otras palabras, las relaciones entre variables sociales son menos estrechas a medida 
que se moderniza un país. Los datos de la Encuesta Mundial de Valores de 1996 lo vuelven a confirmar. Así, por ejemplo:

1. La relación entre el nivel educativo y el tamaño del hábitat en Estados Unidos es más laso (coef. de contingencia $=0,10 ; \mathrm{P}<0,001$ ) que en España (coef. de contingencia $=0,16 ; \mathrm{P}<0,001)$.

2. En Estados Unidos la relación entre el tipo de trabajo (manual y no manual) y el nivel de ingresos familiar es menos estrecha (coef. de contingencia $=0,25$; $\mathrm{P}<0,001$ ) que en España (coef. de contingencia $=0,34 ; \mathrm{P}<0,001$ ).

3. Algo parecido ocurre con la relación entre el tipo de trabajo (manual y no manual) y el nivel educativo: en Estados Unidos es menos fuerte (coef. de contingencia $=0,39 ; \mathrm{P}<0,001$ ) que en España (coef. de contingencia $=0,47 ; \mathrm{P}<0,001$ ).

Se trata de tres ejemplos de cómo las categorías sociales pierden nitidez a medida que avanza la modernización. La hipótesis mantenida en este trabajo es que tal falta de nitidez en el nivel social desdibuja también la relación de la estructura social con el nivel cultural. Los intereses propios de las categorías sociales dejan de estar tan claros. Por lo tanto, disminuye también la proporción de varianza de las respuestas sobre valores, explicada por la estructura macrosocial. Y así ocurre, por ejemplo, en los casos que se exponen a continuación, en los que se analiza la relación entre la media de las respuestas a la pregunta sobre valores ya conocida (v125: preferencia entre igualdad de salarios frente a incentivos salariales), y diversas variables propias de la estructura social:

1. Los cuatro niveles educativos explican la variable dependiente más en España ( $3 \%$ de varianza; coef. $F=13,1 ; P<0,001)$ que en Estados Unidos, en donde los resultados incluso apenas son significativos $(0,4 \%$ de varianza; coef. $\mathrm{F}=3,6 ; \mathrm{P}=0,12$ ).

2. Los tres niveles de ingresos explican la variable en cuestión más en España $(2 \%$ de varianza; coef. $\mathrm{F}=8,2 ; \mathrm{P}<0,001)$ que en Estados Unidos $(0,4 \%$ de varianza; coef. $\mathrm{F}=4,1 ; \mathrm{P}=0,19)$.

3. El tipo de trabajo (manual o no manual) explica más la variable en cuestión en España (coef. $F=14,3 ; \mathrm{P}<0,001$ ) que en Estados Unidos (coef. $\mathrm{F}=0,6 ; \mathrm{P}=0,421$ ).

4. Las trece categorías ocupacionales utilizadas en la Encuesta Mundial de Valores no explican gran cosa de la variable considerada en ambos países, debido, seguramente, al amplio número de categorías empleado en la variable independiente. Sin embargo, se aprecia cierta mínima diferencia: las categorías ocupacionales explican levemente más en España (0,5\% de la varianza; coef. $\mathrm{F}=2,0 ; \mathrm{P}=0,2)$ que en Estados Unidos $(0,1 \%$ de varianza; coef. $\mathrm{F}=1,99 ; \mathrm{P}=$ $0,02)$.

5. Sólo la variable tamaño del hábitat, de las tenidas aquí en cuenta, presenta datos inversos, pero en una proporción tan exigua que no merecen mucha atención. En España estos resultados carecen de significación estadística $(0,0 \%$ de varianza explicada; coef. $\mathrm{F}=1 ; \mathrm{P}=0,367)$. En Estados Unidos los datos tienen poco más de interés $(0,3 \%$ de varianza; coef. $\mathrm{F}=4,79 ; \mathrm{P}=0,008)$. 
Salvo en tres oportunidades, la distribución de las respuestas a la variable analizada (v125) se presenta polarizada, es decir, con curtosis negativa, en casi todas y cada una de las categorías de las variables independientes, tanto en España como en Estados Unidos. No obstante, se aprecia, en general, que la curtosis negativa de los datos de Estados Unidos es levemente inferior a la de España. Es decir, que los datos no están tan polarizados como en nuestro país. El coeficiente de curtosis de la pregunta analizada (v125) en Estados Unidos es $-0,947$ y en España -1,132. En casi todas las categorías analizadas se repite la misma tendencia.

Las excepciones a la curtosis negativa se producen en tres categorias de las trece que tiene la variable independiente denominada categorías ocupacionales. Dos veces en los datos de Estados Unidos (en las categorías de "jornaleros del campo" y "fuerzas armadas"), y una vez en los de España (en la categoría de "capataces"). Por su parte, la distribución de los datos de la variable "ingresos" presenta curtosis positiva en España, y negativa en Estados Unidos, en la mayoría de las categorias de las variables independientes observadas.

Semejante información conduce a tres conclusiones. La primera conclusión es que se revalida la hipótesis secundaria de este trabajo: la polarización en una sociedad desarrollada (Estados Unidos de América) se basa más en la interacción cotidiana y se produce más entre individuos que en una sociedad menos desarrollada (España). La segunda es que la mayor parte de la polarización en todos los países, con independencia del grado de desarrollo, se produce en el nivel micro (en la interacción cotidiana) y mucho menos en el macro, por dos razones: porque el coeficiente de curtosis es negativo pero inferior a $-1,3$ y porque la dependencia de la estructura social es muy baja en cualquier caso. Si la polarización proviniera de causas objetivas y propias de la estructura social de cada país, la curtosis sería mucho más acusada y se marcarían claramente las líneas de fractura social, cosa que no ocurre.

La tercera consecuencia, por último, es que la polarización disminuye cuando la estructura social deja de provocar polarización. En otras palabras, la polarización en el nivel micro no parece compensar del todo la falta de polarización del nivel macro.

\section{CONCLUSIONES}

Según la definición habitual de cultura arbitraria, el comportamiento humano guiado por ella es imprevisible y totalmente aleatorio. No obstante, hemos buscado un orden previsible en el funcionamiento de la misma a partir de la idea de que su funcionamiento se debe a la necesidad humana de distinción e identificación. De ser cierto este mecanismo, el comportamiento dirigido por la cultura arbitraria estaría fundamentalmente polarizado. 
La polarización debida a la cultura arbitraria en una sociedad, sobre todo cuando ésta es moderna, no puede ser demasiado acusada. La propia lógica de la polarización impide la existencia de claras divisiones de la población: la polarización se produce en todos los niveles de sociabilidad, desde el micro (vida social cotidiana) al macro (las clases y otras categorías sociales), compensando unos niveles con otros. Se produce también entre una actitud moderada y otra radical.

Puesto que los valores reúnen las características propias de la cultura arbitraria, hemos acudido a la encuesta mundial de valores de 1995-96 para revalidar la hipótesis de trabajo: las respuestas a preguntas sobre valores presentan un aspecto polarizado en mayor proporción que las respuestas a otras preguntas que no son sobre valores.

El indicador de polarización que hemos usado es el coeficiente de curtosis. La curtosis es igual a cero cuando la distribución de las respuestas se acerca a la distribución normal. Es negativo cuando la distribución es más plana que la normal, es decir, que hay ya indicios de polarización. Es positiva cuando la distribución es más apuntada que la distribución normal, es decir, que hay indicios de acuerdo sobre el tema.

Se ha comparado respuestas a preguntas sobre valores con respuestas a preguntas de hecho (ingresos, años de escolarización y satisfacción con la vida). Los resultados indican que la polarización es una cualidad inherente a las respuestas a preguntas sobre valores, mientras que en los demás tipos de preguntas se puede presentar o no.

Se deduce, asimismo, que la distinción y la identificación se producen y reproducen sobre todo en el nivel microsocial, dado que el coeficiente de curtosis ronda valores bajos $(-1,3)$ : el coeficiente de curtosis sería más elevado (cercano al -2) si la polarización estuviera ligada a categorías propias de la estructura social. Asimismo, en los países más desarrollados el nivel de sociabilidad micro tiene aún más interés que en los menos desarrollados, puesto que en éstos se aprecia mayor dependencia de las respuestas respecto a la estructura social, pese a que el grado de curtosis es muy semejante al de los países más desarrollados.

\section{BIBLIOGRAFÍA}

ALEXANDER, J.C. (ed.) (1989), Durkheimian sociology: cultural studies, Cambridge, Cambridge University Press.

ALEXANDER, J.C.(1989), “Introduction: Durkheimian sociology and cultural studies today”, en ALEXANDER (ed.), Durkheimian sociology: cultural studies, Cambridge, Cambridge University Press, pp. 1-22.

ANDRÉS ORIZO, F. (1996), Sistema de valores en la España de los 90, Madrid, CIS.

BERGER, P.L. (ed.) (1999), Los limites de la cohesión social. Conflictos y mediación en las sociedades pluralistas, Barcelona, Círculo de Lectores. 
RIS

REVISTA INTERNACIONAL DE SOCIOLOCIA

No 37, ENERO-ABRIL, 2004

RAFAEL GOBERNADO ARRIBAS

BERICAT, E. (1989), "Cultura productiva y desarrollo endógeno. El caso andaluz", Revista de Estudios regionales, 24, pp. 15-43.

BOUDON, R. (1979), Effets pervers et ordre social, París, P.U.F.

(1981), La lógica de lo social, Madrid, Rialp.

BOURDIEU, P. (1988), La distinción, Madrid, Taurus (1998 [1979].

BOURDIEU, P. y J.C. PASSERON (1981 [1970]), La reproducción, Barcelona, Laia [1ª, 1970].

CHISSOM, B.S. (1970), "Interpretation of the Kurtosis Statistic", American Statistician, 24, pp. 19-22.

DARLINGTON, R.B. (1970), “Is Kurtosis Really 'Peakedness'?, American Statistician, 24, pp. 19-22.

DÍEZ NICOLÁS, J. y R. INGLEHART (ed.) (1994), Tendencias mundiales de cambio en los valores sociales y politicos, Madrid, Fundesco.

DIMAGGIO, P., J. EVANS y B. BRYSON (1996), “Have Americans' Social Attitudes Become More Polarized?", American Journal of Sociology, 3, nov. pp. 690-755.

DURKHEIM, E. (1973), De la división del trabajo social, Buenos Aires, Schapire [1ª , 1893]

(1982), Las formas elementales de la vida religiosa, Madrid, Akal [1 $\left.1^{\mathrm{a}}, 1912\right]$.

EISENSTADT, S.N. (1992), "Estudios de modernización y teoría sociológica", en CARNERO ARBAT, T. (ed.), Modernización, desarrollo politico y cambio social, Madrid, Alianza, pp. 71-100.

(1992), "The Order-maintaining and Order-transforming Dimensions o Culture", en MÜNCH, R. y SMELSER, N.J.: Theory of Culture, Berkeley, University of California Press, pp. 64-87.

ESCOBAR, M. (1998), "Desviación, desigualdad, polarización: medidas de la diversidad social”, Revista Española de Investigaciones Sociológicas, 82, pp. 9-36.

FERNANDEZ-ABASCAL, H. et al. (1994), Cálculo de probabilidades y estadistica, Barcelona, Ariel.

FERRATER MORA, J. (1979), Diccionario de filosofia, T. 4, Madrid, Alianza

FRONDIZI, R. (1972), ¿Qué son los valores?, Méjico, F.C.E. [1 $\left.1^{\mathrm{a}}, 1958\right]$

GOBERNADO ARRIBAS, R. (2002a), "Los sistemas de identidades: un punto de vista cultural", en VV.AA., La sociedad: teoría e investigación empírica. Libro homenaje a José Jiménez Blanco, Madrid, CIS, pp. 875-890.

(2002b), “Análisis de la adscripción a valores”, Revista Internacional de Sociologia, 33 
INGLEHART, R. (1991), El cambio cultural en las sociedades occidentales avanzadas, Madrid, C.I.S.

KAIERO URÍA, A. (ed.) (1994), Valores y estilos de vida de nuestra sociedad en transformación, Bilbao, Universidad de Deusto.

KERHOFS, J. (1994), "Evolución de los valores en Europa entre 1980 y 1990", en KAIERO URÍA, A. (ed.), Valores y estilos de vida de nuestra sociedad en transformación, Bilbao, Universidad de Deusto, pp. 43-56.

LÓPEZ-CASERO, F., W.L. BERNECKER y P. WALDMANN (comps.) (1994), El precio de la modernización. Formas y retos del cambio de valores en la España de hoy, Madrid, Iberoamericana.

MARIAS, J. (1980), Historia de la filosofia, Madrid, Rev. de Occidente

MÜNCH, R. y N.J. SMELSER (1992), Theory of Culture, Berkeley, University of California Press.

PARSONS, T. (1976), El sistema social, Madrid, Rev. de Occidente (1976 [1951]).

PERRY, R.B. (1954), Realms of Value. A critique of Human Civilization, Cambridge, Mass., Harvard University Press.

PINO ARTACHO, J. y E. BERICAT ALASTUEY (1998), Valores sociales en la cultura andaluza Encuesta Mundial de Valores, Andalucía 1996, Madrid, C.I.S.

ROKEACH, M. (1979), Understanding Human Values. Individual and Societal, Nueva York, The Free Press.

ROS, M. (2002), "Los valores culturales y el desarrollo socioeconómico: una comparación entre teorías culturales", Rev. Española de Investigaciones Sociológicas, $\mathrm{n}^{\circ}$ 99, pp. 9-33.

SANCHEZ CARRIÓN, J.J. (1995), Manual de análisis de datos, Madrid, Alianza Universidad Textos.

SCHMID, M. (1992), "The Concept of Culture and its Place within a Theory of Social Action. A critique of T. Parsons' Theory of Culture", en R. MÜNCH y N.J. SMELSER, Theory of Culture, Berkeley, University of California Press, pp. 88-120.

SIERRA BRAVO, R. (1998), Técnicas de investigación social. Teoria y ejercicios, Madrid, Paraninfo.

SMELSER, N.J. (1971), "Mecanismos de cambio y ajuste al cambio", en B.F. HOSELITZ y W.E. MOORE, Industrialización y sociedad, Madrid, Euramérica, pp. 45-75.

(1992), "Culture: Coherent or Incoherent", en R. MUNCH y N.J. SMELSER (eds.), Theory of Culture, Berkeley, University of California Press, pp. 3-28.

SMITH, C.L. (1991), "Measures and Meaning in Comparisons of Wealth Equality", Social Indicators Research, 24, pp. 367-392. 
RIS

REVISTA INTERNACIONAL DE SOCIOLOGIA

N 37, ENER0-ABRIL, 2004

RAFAEL COBERNADO ARRIBAS

TORCAL LORIENTE, M. (1992), “Análisis dimensional y estudio de valores: El cambio cultural en España”, Revista Española de Investigaciones Sociológicas, 58, pp. 97-122.

TORCAL LORIENTE, M. y P. CHHIBBER (1995), "Elites, cleavages y sistema de partidos en una democracia consolidada: España (1986-1992)", Revista Española de Investigaciones Sociológicas, 69, pp. 7-38.

TREIMAN, D.J. (1970), "Industrialization and Social Stratification”, en E.O. LAUMANN (ed.), Social Stratification: Research and Theory for the 1970s, Nueva york, The Bobbs-Merril Company, pp. 207-234.

WILLIAMS, R.M. (1977 [1968]), “Valores”, en D.L. SILLS (dir.), Enciclopedia Internacional de las Ciencias Sociales, vol. 10, pp 607-610 [1", 1968].

(1979), "Change and Stability in Values and Value Systems: a Sociological Perspective”, M. ROKEACG, Understanding Human Values. Individual and Societal, Nueva York, The Free Press, pp. 15-46. 